\title{
VIVÊNCIA DO ESTUDANTE DE NUTRIÇÃO NA ATENÇÃO PRIMÁRIA: UM RELATO DE CASO
}

\author{
Janilson Avelino da Silva \\ Universidade Federal da Paraíba \\ janilsonsilva@globomail.com \\ Jailane de Souza Aquino \\ Universidade Federal da Paraíba \\ lalaaquino@hotmail.com
}

\section{Resumo}

O propósito deste trabalho é discutir as vivências dos estudantes de Nutrição na Atenção Primária à Saúde-APS que contribuem no processo de sua formação. Método: foram descritos os contatos com a APS por meio da atuação nas Unidades de Saúde da Família-USFs do Município de João Pessoa, Paraíba, durante um período de 3 anos (2010-2012). As principais atividades desenvolvidas pelo estudante de Nutrição foram orientação nutricional, palestras e visitas domiciliares. Resultados: o estudante de Nutrição tem inúmeras possibilidades de atuar na esfera da Saúde Coletiva. A orientação nutricional foi realizada de forma qualitativa devido ao curto tempo de atendimento nas USFs. Os principais temas abordados nas palestras foram os benefícios das frutas e verduras e suas especificidades, soja e a saúde da mulher, alimentação saudável, amamentação, aleitamento materno e afins. As visitas domiciliares ocorreram principalmente a pacientes que estavam incapacitados de locomoção. Conclusão: o relato proporcionou uma análise das possibilidades de atuação do estudante de Nutrição nas USFs pautadas nos princípios e diretrizes do Sistema Único de Saúde-SUS, visando uma melhor atuação na rede de assistência pública à saúde.

Palavras-chave: Saúde Pública; Nutricionista; Programa Saúde da Família.

\section{EXPERIENCE STUDENT NUTRITION IN PRIMARY CARE: A CASE REPORT}

\begin{abstract}
The proposal of this article is to discuss the experiences of the students Nutrition in Primary Health Care APS-contributing in their training process. Method: contacts with APS through work in the Family Health Units-USFs the Municipality of João Pessoa, Paraiba were described over a period of three years (2010-2012). The main activities undertaken by the student were Nutrition nutritional counseling, home visits and lectures. Results: Student Nutrition has numerous possibilities to act in the sphere of Public Health. Nutritional counseling was conducted qualitatively due to short service time in USFs. The main topics
\end{abstract}

\section{Esta obra está licenciada sob uma Licença Creative Commons.}


covered in the lectures were the benefits of fruits and vegetables and their specificities, soy and women's health, healthy eating, breastfeeding, breastfeeding and the like. Home visits were mainly to patients who were incapable of locomotion. Conclusion: The report provided an analysis of the possibilities of student performance in Nutrition USFs guided by the principles and guidelines of the National Health System-SUS, to improve performance in the public health care

Keywords: Public Health; nutritionist; Family Health Program.. 


\section{囟 INTRODUÇÃ̃O}

UNIVERSIDADE FEDERAL DE SANTA CATARINA

PRÓ-REITORIA DE CULTURA E EXTENSÃO

DEPARTAMENTO DE PROJETOS DE EXTENSÃO

EXTENSIO - REVISTA ELETRÔNICA DE EXTENSÃO

O Sistema Único de Saúde (SUS) detém três níveis de atenção à Saúde que devem funcionar de forma integrada, cada um tendo seu papel bem definido, embora com o objetivo comum de produzir saúde. A atenção primária à saúde, porta de entrada do SUS, dispõe de profissionais de teor mais generalista, voltados à promoção e prevenção de doenças nas Unidades de Saúde da Família. Tal setor tem sido relevante em situações de enfrentamento de insegurança alimentar e nutricional. Estudantes do curso de Nutrição deverão obter formação abrangente, incluindo esses aspectos (MELO, ALCHIERI e NETO, 2012).

Há um número reduzido de nutricionistas neste nível de atenção, implicando muitas das vezes no encaminhamento da população a outros níveis de atenção à saúde. A atuação do nutricionista na Atenção Primária é justificada como forma de garantir a qualidade e a integralidade da atenção à saúde. As mudanças no perfil de morbimortalidade da população refletem-se no processo de transição nutricional requerendo profissionais capazes de auxiliar na prevenção e agravos à saúde e os desfechos ocasionados pela transição nutricional, sendo importante uma ampliação da atuação do profissional de nutrição na Estratégia Saúde da Família para melhor resolver e maximizar a capacidade de resolução destes problemas (CFN, 2008).

Segundo Assis et al. (2002), a competência do nutricionista para integrar a equipe da Estratégia Saúde da Família está estabelecida em sua formação acadêmica, a qual o instrumentaliza a realizar o diagnóstico nutricional da população, tornando-o, assim, o único profissional a receber uma instrução específica que lhe permite, a partir desse diagnóstico e da observação dos valores socioculturais, propor orientações dietéticas cabíveis e necessárias, adequando-as aos hábitos da unidade familiar, à cultura, às condições fisiológicas dos grupos e à disponibilidade de alimentos. Trata-se, portanto, de um profissional apto a participar efetivamente da recriação das práticas de atenção à saúde no Brasil.

O nutricionista tem inúmeras possibilidades de atuar na esfera da Saúde Coletiva como, na atenção individual, na atenção em grupos, nas atividades de auxílio à coordenação e execução de programas, na realização de diagnósticos alimentares, na avaliação da situação de segurança alimentar do grupo, em ações de promoção de alimentação saudável, na vigilância nutricional e, ainda, em pesquisa. Nesse contexto, o estudante de Nutrição inserido na Saúde Pública pode auxiliar nessas atividades, visto que seu currículo se demonstra tão 


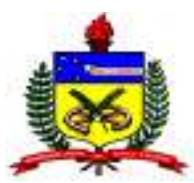

UNIVERSIDADE FEDERAL DE SANTA CATARINA

PRÓ-REITORIA DE CULTURA E EXTENSÃO

DEPARTAMENTO DE PROJETOS DE EXTENSÃO

EXTENSIO - REVISTA ELETRÔNICA DE EXTENSÃO

abrangente que o qualifica para tal exercício (PINHEIRO et al, 2012). Ademais, a articulação entre o ensino na graduação e as ações de extensão é de grande importância para o estudante, pois se podem construir conhecimentos, aliando o aprendizado teórico da sala de aula ao contato prático com a realidade da comunidade, complementando e ampliando seu aprendizado, transcendendo a formação meramente técnico-profissional (PINHEIRO et al. ,2012)

Sabendo-se das inúmeras possibilidades de atuação no nível primário de assistência à saúde, se faz necessário discutir cada vez mais a prática de estudantes de Nutrição nas Unidades de Saúde, visto que os estudos que tratam dessa temática são escassos. Este relato objetiva discutir sobre as vivências dos estudantes de Nutrição na Atenção Primária à SaúdeAPS que contribuem no processo de sua formação.

\section{MATERIAL E MÉTODOS}

Todos os contatos com a Atenção Primária à Saúde por meio da atuação nas Unidades de Saúde da Família-USFs do Município de João Pessoa- P.B., sendo algumas integradas, ocorreram durante um período de 3 anos (2010-2012) e foram reunidos para embasar a escrita deste relato. Foram incluídas atividades de projetos e estágios realizados durante a graduação do curso de Nutrição da Universidade Federal da Paraíba-UFPB. Para descrição das vivências foram utilizados diários de estágio compostos por anotações das ocorrências relacionadas à Nutrição nas USFs durante toda atuação. Os diários eram escritos por um estudante de graduação em Nutrição e os mesmos eram dotados de data, localização, descrição e percepção das atividades.

As atividades selecionadas para esse relato foram as mais frequentes nas USFs como a orientação nutricional, as palestras e as visitas domiciliares. Embora, outras atividades tenham sido realizadas como ações de saúde na comunidade, reuniões de equipe, feiras, dentre outras.

Todos os atendimentos para orientação nutricional foram registrados como forma de facilitar os retornos. As principais informações registradas foram: nome, idade, peso, altura, circunferência da cintura, doença pré-existente e medicação utilizada. Para auxiliar nos atendimentos o estudante organizou previamente todos os materiais necessários para atendimento de todas as faixas etárias, como equações, tabelas, calculadora e material informativo, para que o atendimento ocorresse com facilidade e eficácia.

\section{cc) (†) (-) Esta obra está licenciada sob uma Licença Creative Commons.}




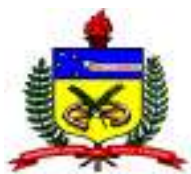

UNIVERSIDADE FEDERAL DE SANTA CATARINA PRÓ-REITORIA DE CULTURA E EXTENSÃO

DEPARTAMENTO DE PROJETOS DE EXTENSÃO

EXTENSIO - REVISTA ELETRÔNICA DE EXTENSÃO

As palestras ocorreram de forma grupal, em que os indivíduos foram organizados em círculo, existindo participação ativa da população, uma vez que a produção do círculo deixa todos os participantes no mesmo nível, visível e acessível a todos do grupo. Após a realização desta atividade era realizada uma avaliação e uma enquete aos participantes sobre as preferências quanto aos temas, visto que a seleção destes pode influenciar no interesse e atenção durante as palestras. Os temas foram transmitidos da forma dinâmica por meio de perguntas, respostas construídas em conjunto e utilização de recursos audiovisuais como vídeos, músicas, cartazes, reálias, jogos e outros recursos.

As visitas domiciliares foram realizadas na companhia de um Agente Comunitário de Saúde-ACS, pois é um membro da Assistência Primária a Saúde que reconhece muitos aspectos da área de atuação e detém contato diário com a população. Durante a atuação nas Unidades os ACS foram elementos-chave ao convidar a população para as atividades nas Unidades de Saúde. As visitas foram realizadas a partir de demanda espontânea da população ou de visitas rotineiras realizadas pelos ACSs.

\section{RESULTADOS}

De maneira geral, os estudantes em comum acordo com a coordenação de estágios podem discutir se irão calcular dietas ou apenas orientar de forma qualitativa os alimentos a serem prescritos. A orientação dietética de forma qualitativa tem sido bastante recorrente devido ao fator tempo e pelo fato de haver grande rotatividade entre os estudantes. Infelizmente, o Nutricionista não se encontra inserido em todas as USFs da Paraíba para prestar assistência contínua aos tratamentos nutricionais. A orientação nutricional é uma ferramenta muito importante na atuação do nutricionista, visto que nele o estudante poderá aplicar todos os conhecimentos adquiridos em sala de aula. Faz-se necessário realizar uma adequação da linguagem levando-se em consideração os aspectos culturais e socioeconômicos dos usuários; se isso não acontecer poderá não se atingir os objetivos da consulta. O estudante irá propor mudanças no padrão alimentar do indivíduo, adequando ao seu estilo de vida.

Muitos recém-nascidos foram encaminhados para o estudante de Nutrição durante a atuação nas Unidades de Saúde. Assim, o mesmo teve de enfrentar muitos obstáculos em relação ao convencimento às mães a respeito da amamentação exclusiva até os seis meses, 


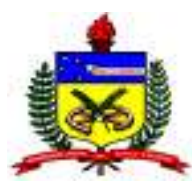

UNIVERSIDADE FEDERAL DE SANTA CATARINA

PRÓ-REITORIA DE CULTURA E EXTENSÃO

DEPARTAMENTO DE PROJETOS DE EXTENSÃO

EXTENSIO - REVISTA ELETRÔNICA DE EXTENSÃO

pois muitas delas exigiam a prescrição de fórmulas lácteas e/ou alimentos em substituição ao leite materno.

A partir do exposto, se faz necessário que haja uma constante leitura e atualização para se adquirir o conhecimento específico da temática, assim como também de um planejamento para que o aconselhamento ocorra de maneira responsável e consciente.

Os temas recorrentes abordados nas palestras foram os benefícios das frutas e verduras e suas especificidades, soja e a saúde da mulher, alimentação saudável, amamentação, aleitamento materno e afins. O estímulo da participação da comunidade assistida pelas USFs é necessário para que haja um maior aprendizado e uma maior probabilidade destes usuários utilizarem os conhecimentos adquiridos no dia a dia.

Outro foco, onde o estudante de Nutrição atuou foi nas visitas domiciliares. Nestas, houve a produção de cuidado nas residências dos indivíduos. Fato significativo, visto que a atuação no ambiente em que o indivíduo vive ocorre de forma mais real, factível. É um espaço de aprendizado amplo, pois não foram utilizados conhecimentos estritos da Ciência da Nutrição e sim outros aspectos que podiam melhorar a Saúde dos usuários.

A maior frequência da solicitação da presença do estudante de Nutrição para as visitas domiciliares esteve relacionada a pacientes que estavam incapacitados de locomoção (exemplo, os que tinham sofrido um Acidente Vascular Cerebral-AVC ou que estavam em estágios difíceis de neoplasias ou ainda cadeirantes), ou aqueles que podiam se locomover, mas que tinham fobia a locais que fizessem alusão a hospitais e ainda aqueles que gostam de comodidade.

\section{DISCUSSÃO}

Pode-se considerar um desafio para o estudante de Nutrição atuar nas Unidades de Saúde, visto que muitas das vezes o profissional de Nutrição não faz parte da equipe de saúde. As ações dependem, majoritariamente da criatividade, força de vontade e criação de vínculo com a população e a Unidade de Saúde. É imperativo observar o indivíduo como um todo, ou seja, considerar a alimentação como um fenômeno psicobiossociocultural. Deve haver um cuidado para que não haja um olhar fragmentado no cuidado Nutricional (DEMÉTRIO; PAIVA; FRÓES et al. 2011).

\section{Esta obra está licenciada sob uma Licença Creative Commons.}




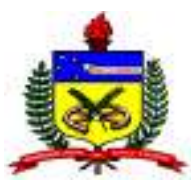

UNIVERSIDADE FEDERAL DE SANTA CATARINA

PRÓ-REITORIA DE CULTURA E EXTENSÃO

DEPARTAMENTO DE PROJETOS DE EXTENSÃO

EXTENSIO - REVISTA ELETRÔNICA DE EXTENSÃO

A atenção nutricional individualizada seguiu as recomendações da Associação Americana de Diabetes (ADA, 1994), em que a assistência dietoterápica deve estar de acordo com os requerimentos nutricionais do indivíduo, incluindo a avaliação do estado nutricional, a identificação dos problemas nutricionais, planejamento de ações dietoterápicas e a avaliação dessa assistência. O estudo de Kwi Ock e Smi (2012) demonstraram que a orientação nutricional individualizada é eficaz no aumento da ingestão de nutrientes quando compararam indivíduos que receberam orientação com os que não receberam orientação assim caracterizada.

As palestras levam Educação Nutricional de forma grupal, entretanto considerando que a alimentação é algo complexo e dotado de diversos significados para os indivíduos (DEMÉTRIO et al. 2011). Então, este aprendizado deve ser construído em conjunto, aproveitando todas as colocações do grupo. Em uma pesquisa realizada no município de João Pessoa sobre Educação em Saúde foi verificado que houve um baixo envolvimento do profissional de Nutrição; de 21 profissionais avaliados, apenas 12 tinham participação ativa (LIMA et al., 2000).

É necessário que haja um maior comprometimento por parte dos profissionais nutricionistas para que haja um maior fortalecimento e reconhecimento da classe, ganhando maior visibilidade junto a outros profissionais de saúde. Ademais, os estudantes de Nutrição que acompanham os profissionais nas Unidades de Saúde terão mais estímulo ao desenvolverem suas atividades de estágio. As palestras, os cartazes e os folhetos explicativos foram os elementos mais utilizados nos processos de educação em Saúde relatado no estudo de Lima, Barros e Melo (2000) assemelhando-se aos recursos utilizados frequentemente nas palestras nas Unidades de Saúde pelos estudantes de Nutrição desta vivência.

As visitas domiciliares ocorreram conforme recomendação do Conselho Federal de Nutricionistas em suas ações estratégicas. Recomenda-se haver a identificação das características dos indivíduos e da família, atuando precocemente diante das dificuldades que possam afetar o estado nutricional, bem como a segurança alimentar e nutricional da família (CFN, 2008). Alguns indivíduos se encontram em processo de internação domiciliar e, às vezes, precisam de orientações quanto à alimentação, seja para indicação de algum suplemento, seja pra modificar algum outro aspecto alimentar ou até mesmo para se articular

\section{Esta obra está licenciada sob uma Licença Creative Commons.}


UNIVERSIDADE FEDERAL DE SANTA CATARINA

PRÓ-REITORIA DE CULTURA E EXTENSÃO

DEPARTAMENTO DE PROJETOS DE EXTENSÃO

EXTENSIO - REVISTA ELETRÔNICA DE EXTENSÃO

com outras instituições (intersetorialidade) visando a melhora do estado de saúde do paciente/usuário (GEUS et al., 2011).

As mudanças do perfil epidemiológico, demográfico e nutricional dos últimos anos, assim como os avanços do conhecimento requerem ações específicas de Nutrição que atendam a demanda da população. O nutricionista deve ter formação para contribuir na garantia da Segurança Alimentar e Nutricional (SAN) da população brasileira, embora Vieira e Cervato-Mancuso (2014), tenham revelado que as universidades ainda não estão preparadas para uma formação embasada nesse contexto. Ademais, ações de Nutrição devem estar integradas às demais ações de saúde nas redes de atenção, tendo a atenção básica como base dessas ações (BRASIL, 2012).

\section{CONSIDERAÇÕES FINAIS}

As reflexões presentes neste estudo permitem afirmar que o estudante de nutrição detém inúmeras possibilidades de atuação no nível primário de assistência à saúde e que estas devem ser bem aproveitadas para o seu processo de formação profissional. Tais atividades devem ser pautadas numa lógica que extrapole a prática biologicista do cuidado para que o profissional formado tenha uma postura diferenciada e uma visão crítica da realidade em que o mesmo está inserido.

Há a necessidade de uma revisão dos atuais programas políticos e pedagógicos de Nutrição dos diversos cursos de graduação, dando ênfase a fatores como carga horária e qualidade de ensino. Mais estudos são necessários para avaliar os impactos dessa formação na saúde da população.

O relato proporcionou uma análise das possibilidades de atuação do estudante de Nutrição nas USFs pautadas nos princípios e diretrizes do Sistema Único de Saúde-SUS, visando uma melhor atuação na rede de assistência pública à saúde.

\section{REFERÊNCIAS}

AMERICAN DIETETIC ASSOCIATION (ADA). Identifying patients at risk: ADA's definitions for nutrition screening and nutritional assessment. J Am Diet Assoc.v. 94, n.8, p. 838-839, 1994.

\section{Esta obra está licenciada sob uma Licença Creative Commons.}




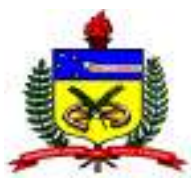

$$
\begin{aligned}
& \text { UNIVERSIDADE FEDERAL DE SANTA CATARINA } \\
& \text { PRÓ-REITORIA DE CULTURA E EXTENSÃO } \\
& \text { DEPARTAMENTO DE PROJETOS DE EXTENSÃO } \\
& \text { EXTENSIO - REVISTA ELETRÔNICA DE EXTENSÃO }
\end{aligned}
$$

ASSIS et al. O Programa Saúde da Família: contribuições para uma reflexão sobre a inserção do nutricionista na equipe multidisciplinar. Rev. Nutr. v.15, n.3, p.255-266, 2002.

BRASIL. Ministério da Saúde. Política Nacional de Alimentação e Nutrição. Brasília, DF, 2012. 88p.

Conselho Federal de Nutricionistas (CFN). O Papel do Nutricionista na Atenção Primária à Saúde. Brasília (DF), p. 1-36, 2008.

DEMÉTRIO et al. A nutrição clínica ampliada e a humanização da relação nutricionistapaciente: contribuições para reflexão. Rev. Nutr. v.24, n.5, p.743-763, 2011.

GEUS et al. A importância na inserção do nutricionista na Estratégia Saúde da Família. Ciência \& Saúde Coletiva.v.16, n.1, p. 797-804, 2011.

KWI OCK; SMI. Effects of Individualized Nutritional Education Programs on the Level of Nutrient Intake and Nutritional Status of Colorectal Cancer Patients Undergoing Palliative Chemotherapy.J Korean Acad Nurs.v.42, n.6, p. 799-809, 2012.

LIMA et al. Educação em Saúde e Nutrição em João Pessoa, Paraíba. Rev. Nutr. v.13, n.1, p. 29-36, 2000.

MELO, ALCHIERI e NETO. Sistema Único de Saúde: uma avaliação realizada em Natal, Rio Grande do Norte, Brasil. Psico-USF. v. 17, n.1, p. 63-72, 2012.

PINHEIRO et al. Percepção de professores e estudante em relação ao perfil de formação de nutricionista em saúde pública.Rev. Nutr.v. 25, n.5, p. 632-643, 2012.

VIEIRA; CERVATO-MANCUSO. Professional training in the context of food and nutrition security. Prim Health Care Res Dev. p. 1-5, 2014.

\section{Esta obra está licenciada sob uma Licença Creative Commons.}

